\title{
Wireless Shelf Life Monitoring and Real Time Prediction in a Supply-Chain of Perishable Goods
}

\author{
V.F. Annese, S. Cipriani, G. Biccario, D. Di Marzio, D. De Venuto \\ Department of electronics and informatics engineering, \\ Politecnico di Bari, Via Orabona 4 - Bari , 70125 , Italy
}

\begin{abstract}
This paper discusses the huge potential of a Wireless Sensor Network (WSN) as a tool for real-time monitoring in a perishable goods supply chain according to the pressing need of security and food certification. The combination of an appropriate monitoring system and further data processing create a tool that can provide the most useful information for each application. In this paper we propose a case study.
\end{abstract}

Keywords-wireless sensor network; safety; food certification; shelf life.

\section{INTRODUCTION}

Nowadays safety and certification for food production continue to be a requirement so strict that global associations such as the FDA ("Food and drug administration") or the WHO ("World Health Organization”) are continuously in activity to promote methods of monitoring and control more and more efficient [1]. The organoleptic properties of food, raw food, cosmetics, drugs, specialty oral use, and others, have a determining effect not only on the safety of the product for human use but also on consumption and commercial success. Hence the need to study, define and evaluate them correctly in order to prevent their earlier degradation. A low power "ad hoc" WSN is a successful solution for real-time monitoring perishable goods. The WSN we used in our demonstrative monitoring underlines the potentiality of the system. The real time data availability, the low power consumption, the possibility of expand the WSN using the most appropriate components for each application, the moderation of the production costs and the adequate precision of the measures are all great reasons that make a WSN an advantageous approach in perishable food supply chain. In fact, the loss of perishable products is estimated at approx. \$35 billion annually all around the world and especially in US [6]. Moreover in the last years high interest in the field of safety and certification in food production has been shown. A multitude of security protocols and certification has been developed and used in all areas of the food supply chain and, among all, the "Hazard analysis and critical control points" (HACCP) method continues to be worldwide increasingly prevalent. HACCP is a scientific and technical approach for the prevention of biological, chemical, and physical hazards, whose seven fundamental principles are released and determined by the standard ISO 22000 [2]. The HACCP aim is to identify the different critical control points (CCPs) in food production and to define the parameters of interest for suitably monitoring every phase of the productive process. This approach creates a major emphasis on food quality, particularly with regard to health and safety, a concept that goes beyond mere customer satisfaction, but rather pointing to the protection of public health [1]. This new approach makes necessary the design of a versatile, efficient and low cost methodology for the real-time monitoring. In this paper, it is proposed a WSN constituted by multisensor nodes monitoring each item or pallet. The solution is not expensive and allow a safe monitoring of several typology of goods. Just for give an estimation of the costs let's try to make a budget statement. If produced in a supply chain, we can estimate the cost of each node of about $€ 15$ (considering nodes of high quality with re-programmability and reusability characteristics) and about $€ 500$ for each gateway. A system made by 3 gateways and 45 sensors (considered sufficient to manage a medium-sized productive environment) would cost about $€ 2,175$. Estimating the lifetime of the gateway (changing individual nodes is not a problem) for about 4 years, we are talking about $€ 544$ annually. These costs would certainly be overcome by the consequent reduction of wastage in perishables chain and the corresponding increase in sales (due to the added value that such a monitoring system can provide the product). The paper is organized as following: the paragraphs 2 and 3 propose as case study a WSN architecture and a demonstrative monitoring of three storage conditions affecting the organoleptic properties of perishable goods, i.e. temperature, humidity and light exposition. The measurements have been realized in a warehouse for storage of agricultural products. The paragraph 4 shows an algorithm for processing of the obtained data: a shelf life estimation through a calculation algorithm based on the Arrhenius law.

\section{WIRELESS SENSOR NETWORKS}

\section{A. Definition and generalities}

A Wireless sensor network (WSN) is an infrastructure composed by wireless nodes, with little memory and a lowperformance CPUs, capable of performing measurements, processing and communicating wirelessly to a central point, where the data are managed. The structure typically involves several wireless scattered nodes in a specific area 
periodically sending the collected data to a coordinator point (gateway), which manages the network and forwards them to another remote system for further processing [7]. The wireless access is usually a "contention-oriented random access" type, as defined in the IEEE 802, but IEEE 802.15.4 is the most commonly used standard (it defines the physical and MAC layers for LoWPAN, wireless personal area networks with low data-rate) and ZigBee. This is due to IEEE 802.15.4 applicative advantages like worldwide defined operative band (2.4 GHz ISM), good data rate (250 kbps) and range of action, low power consumption, possibility of routing and retransmission in case of errors. The OSI model can describe a WSN even if layer separation in a WSN is not so clear: the layers overlap each other in order to create efficient communication protocols, advanced power saving features and network flexibility. The second and third layer are the core of the network: they deal with solving the difficult challenges in a wireless sensor network just like scalability and communication problems. The physical layer plays a fundamental role, without whom the network would not even exist. The communication level, if implemented, is not defined by specific rules and, in any case, is not complicated as in the wired network TCP / IP. The levels 5, 6 and 7 are usually valid only for gateways or for hosts. ZigBee and other OEM solutions make exceptions networks, which also implement the application layer nodes [8].

\section{$B$. Architecture of a light / temperature / relative humidity WSN}

The WSN used for the monitoring activity is made up of six nodes to obtain information about the environmental light, temperature and humidity (figure 2). Each unit can process these data and send them to a coordinator whose task is to make the received data available in real time (apart from the communication delays). The communication between each node and gateway is wireless using Zigbee protocol. Subsequently, the gateway upload the data to a cloud using an internet connection. The real-time data are available on the web using a tablet, a smartphone, a PC or any device with internet connectivity by logging in the data cloud that manages the data. An appropriate authentication system allows access only to authorized users. In figure 2 there is a demonstrative diagram about the overall architecture of a single node.The small size $(6.85 \mathrm{~cm} \times 6.35$ $\mathrm{cm} \times 3.30 \mathrm{~cm})$ and the independent power supply allow proper positioning of the sensors. Due to the use of the Zigbee technology and to its low power consumption, the battery life is durable. The following table (Table I) summarizes the main performance of each node. The analog outputs of the sensors are digitized by a 12bit resolution ADC and then are processed for transmission.

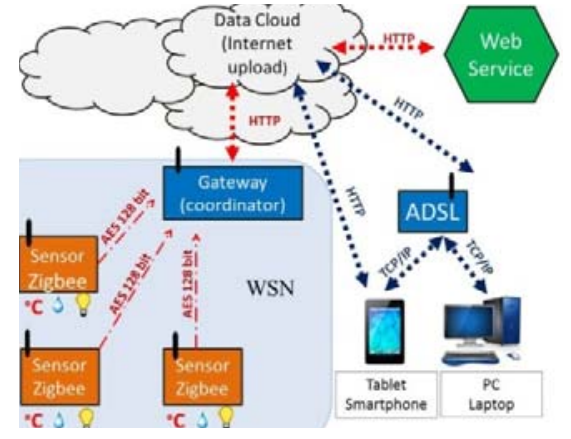

Figure 1. Architecture of a WSN

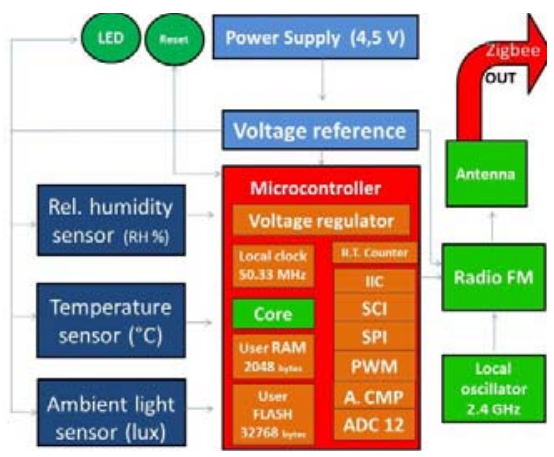

Figure 2. Architecture of a WSN node

TABLE I. SENSOR PERFORMANCES.

\begin{tabular}{|c|c|c|}
\hline & Specification & Value \\
\hline \multirow{3}{*}{ Power supply } & Read-sleep cycle/ battery & 1 per 30s/ $1.5 \mathrm{y}$ \\
\hline & life & 1 per $60 \mathrm{~s} / 2.5 \mathrm{y}$ \\
\hline & AC input V & $3 \times$ AA $1.5 \mathrm{~V}$ battery \\
\hline \multirow[b]{2}{*}{ Temp. sensor } & Range & -18 to $+55^{\circ} \mathrm{C}$ \\
\hline & Accuracy & $+/-2$ \\
\hline \multirow{3}{*}{$\begin{array}{l}\text { Ambient } \\
\text { Light sensor }\end{array}$} & Bandwidth Range & 360 to $970 \mathrm{~nm}$ \\
\hline & $\begin{array}{c}\text { Wavelength of peak } \\
\text { sensitivity }\end{array}$ & $570 \mathrm{~nm}$ \\
\hline & Luminance range & $\begin{array}{c}10 \text { to } 1000 \text { lux }(+/- \\
20 \%)\end{array}$ \\
\hline \multirow{3}{*}{$\begin{array}{c}\text { Relative } \\
\text { humidity sensor }\end{array}$} & Range & 0 to $95 \% \mathrm{RH}$ \\
\hline & Interchangeability & $\begin{array}{c}+/-5 \%(0 \text { to } 59 \% \\
\text { RH) } \\
+/-8 \%(60 \text { to } 95 \% \\
\text { RH) }\end{array}$ \\
\hline & Accuracy & $+/-3.5 \% \mathrm{RH}$ \\
\hline \multirow{3}{*}{$\begin{array}{c}\text { Zigbee } \\
\text { transmission }\end{array}$} & RF date rate & $250 \mathrm{kbps}$ \\
\hline & Frequency & ISM $2.4 \mathrm{GHz}$ \\
\hline & Indoor/line of sight range & $40 \mathrm{~m} / 120 \mathrm{~m}$ \\
\hline
\end{tabular}

III. WSN IN PERISHABLE GOODS SUPPLY-CHAIN: EXPERIMENTAL RESULTS

In the following a case study is described to demonstrate the feasibility and effectiveness of the proposed solution. The ad-hoc solution requires a calibration of the smart sensor selected once inside the "working" environment and positioning and mapping phase of the sensor to get the tracking of the product. 
A. Working verification through a reference given by precision instruments

Using comparative reference, we measured sensors precision. During just one hour session we acquired 20 samples and we compared them with more accurate instruments (luxmeter: Yokogawa 510 O2; environmental hygrometer - thermometer : Testo 608-H1). The measurements were carried out indoor with artificial lights. The graphs obtained (figure 3 - data in table II), where the red dashed line represents the reference, demonstrate that each sensor is sufficiently stable (rising and falling edge are followed) in a known and modest range of values. The sensors accuracy is sufficient for an environmental monitoring which therefore does not require extreme precision.
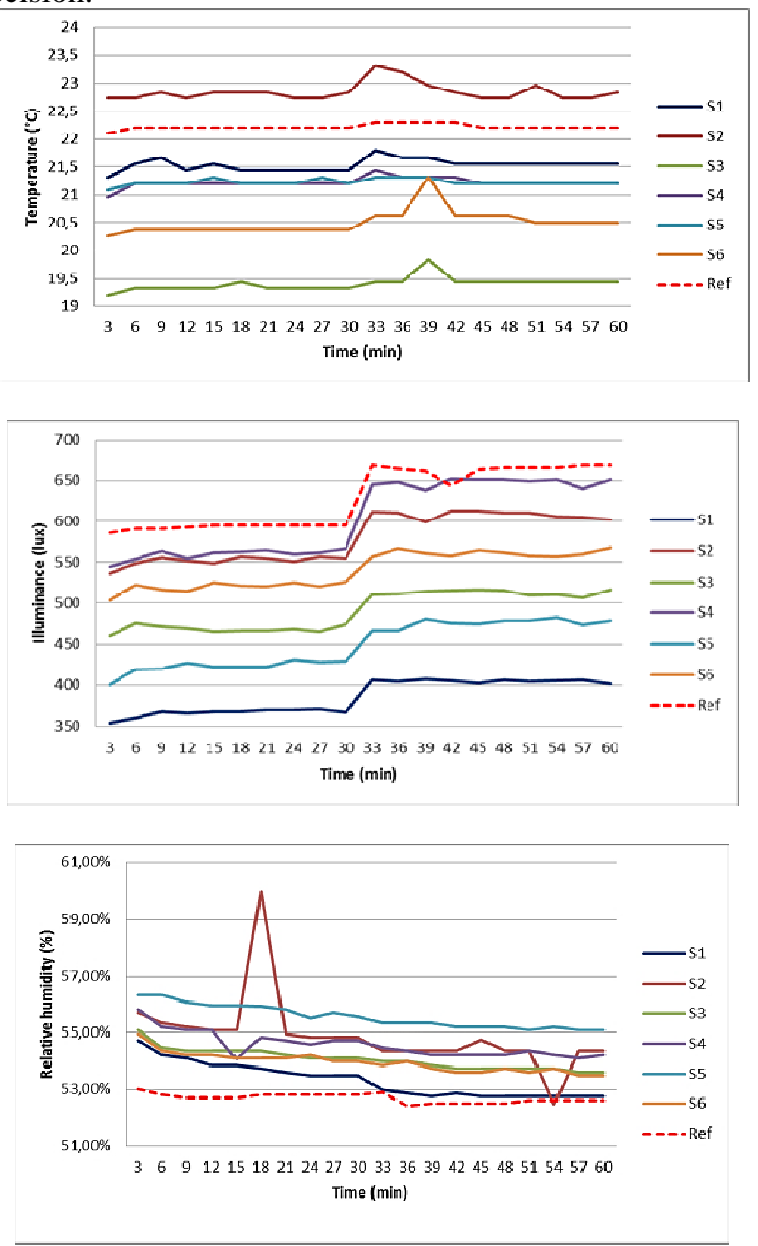

Figure 3. Sensors calibration.
TABLE II. MEASURED DATA

\begin{tabular}{|c|c|c|}
\hline & Average $[\mathrm{lx}]$ & StdDev [lx] \\
\hline S1 & 385.6 & 19.78 \\
\hline S2 & 580.05 & 28.73 \\
\hline S3 & 491.05 & 22.48 \\
\hline $\mathrm{S} 4$ & 603.65 & 44.30 \\
\hline S5 & 449.45 & 27.47 \\
\hline S6 & 540.75 & 21.24 \\
\hline \multirow[t]{2}{*}{ Ref } & 628.1 & - \\
\hline & Average $\left[{ }^{\circ} \mathrm{C}\right]$ & StdDev $\left[{ }^{\circ} \mathrm{C}\right]$ \\
\hline $\mathrm{S} 1$ & 21.54 & 0.11 \\
\hline $\mathrm{S} 2$ & 22.84 & 0.16 \\
\hline S3 & 19.05 & 0.12 \\
\hline $\mathrm{S} 4$ & 21.21 & 0.086 \\
\hline S5 & 21.22 & 0.06 \\
\hline S6 & 20.50 & 0.22 \\
\hline \multirow[t]{2}{*}{ Ref } & 22,21 & - \\
\hline & Average rh[\%] & StdDev [\%] \\
\hline S1 & 53.32 & 0.0060 \\
\hline $\mathrm{S} 2$ & 54.89 & 0.0132 \\
\hline S3 & 54.06 & 0.0036 \\
\hline S4 & 54.57 & 0.0045 \\
\hline S5 & 55.56 & 0.0041 \\
\hline S6 & 53.95 & 0.0035 \\
\hline Ref & 52.68 & - \\
\hline
\end{tabular}

B. Demonstrative warehouse monitoring using a real time light-T-Rh WSN

We used the above WSN for a demonstrative example of warehouse monitoring in which were stored agricultural products. Figure 4 shows the measurement results: the temperature, relative humidity and light exposition have been monitored for several hours. Three multisensor modules have been used to monitor three different pallets in the same environment. Each stock was monitored for 5 hours, from 10:17 to 15:17. In particular, three sensors were placed: one in the box items (blue curve in figure 4), the others outside of it. The data collected and stored were available to any authorized user in the world in real time. Thanks to the battery life durability, it would be possible a monitoring of the entire food chain it in the same way. This would promote transparency in the food chain, becoming a guarantee for the consumer, a powerful low-cost tool for the producer and a simple control method for the organs predisposed. 

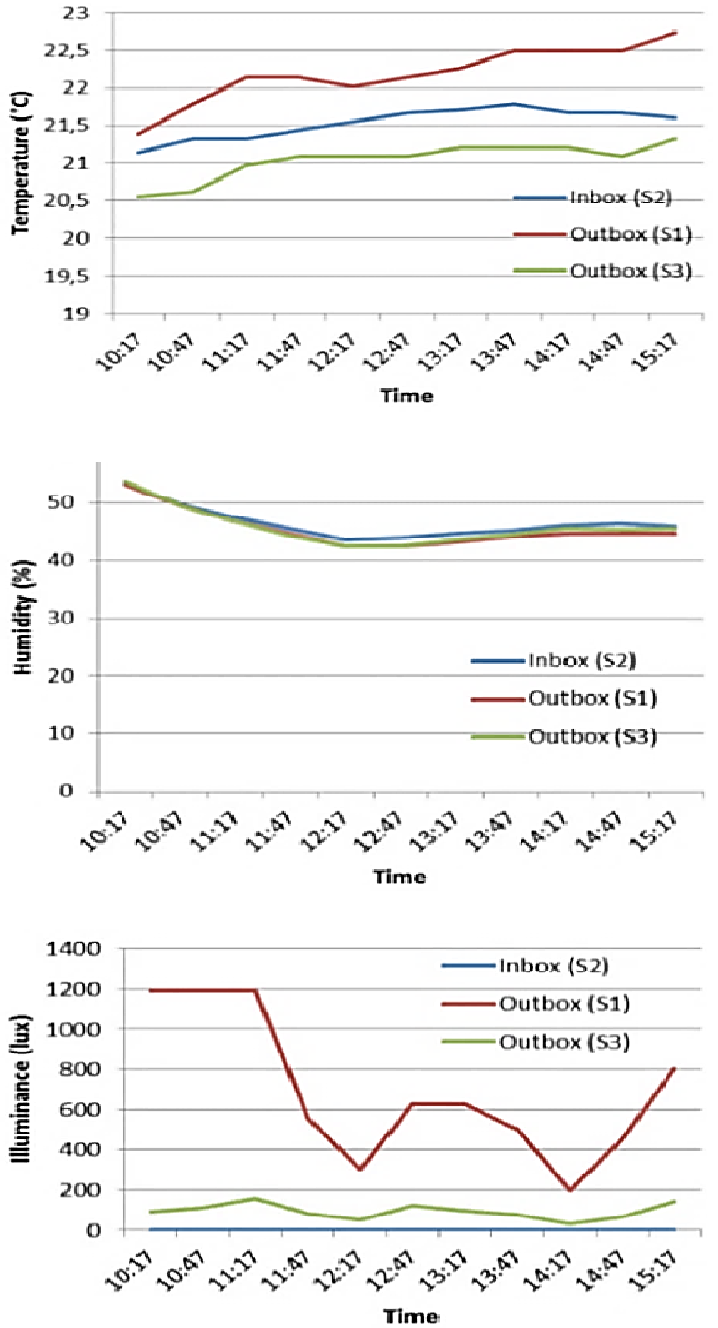

Figure 4. Five hours pallet monitoring results (from the left top, clockwise: $\mathrm{T}, \mathrm{Rh}$, lux).

\section{SHELF LIFE AND QUALITY DEGRADATION RATE ESTIMATION}

Shelf life is the period of time in during which a perishable product may be stored without becoming unhealthy for use or consumption. We propose an implementation of Arrhenius and Lambert law in a calculation algorithm to estimate the shelf life and the degradation rate of the products monitored using the WSN whose characteristics are known in a standard situation.

\section{A. Linear mathematical model of quality degradation prediction}

In this treatment, we suppose linear inversely proportionality between quality and time(figure 5): C stands for the food quality (Co: best condition ; Ce: minimum limit of quality), ts for the shelf time, $\mathrm{K}$ for the quality degradation speed. $\mathrm{K}$ depends on the temperature according to Arrhenius law [4] :

$$
\frac{d C}{d t}=K(T)=W e^{\frac{-E a}{R T}}
$$

Co, Ce, ts , To have to be considered known (best situation). Ko results:

$$
K_{o}=\frac{C_{e}-C_{o}}{t_{s}} .
$$

If at time $\mathrm{t} 1$ temperature rises up to $\mathrm{T} 1>\mathrm{To}$, the shelf life decrease. It is possible to estimate $\mathrm{K} 1$ (the new degradation speed) using the eq. (1). The new perspective will produce a new lower shelf time ts1. The new

characteristic can be expressed as:

$$
C=k_{1} \cdot t+\frac{C_{1}}{K_{1} \cdot t_{1}} .
$$

If at ts temperature returns to To, the shelf life must not change: by now the

product is compromised, applying the prevention principle.

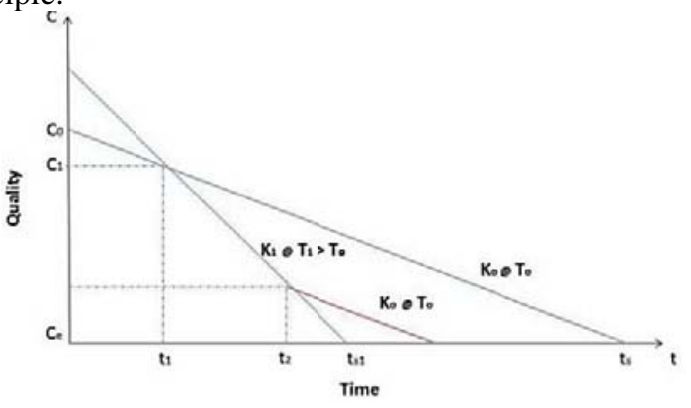

Figure 5. Linear model of quality degradation

\section{B. Presentation of a calculation algorithm for the shelf life estimation}

The used calculation algorithm is here presented with a flow chart (figure 6) that implements the model described above. The purpose is to create an application for smartphone and tablet which is able to calculate in real time how long the product preserve the quality specifications in that environment. The algorithm enables a real time prevision of the remaining shelf time of a perishable product: it allows an immediate preventive intervention. 


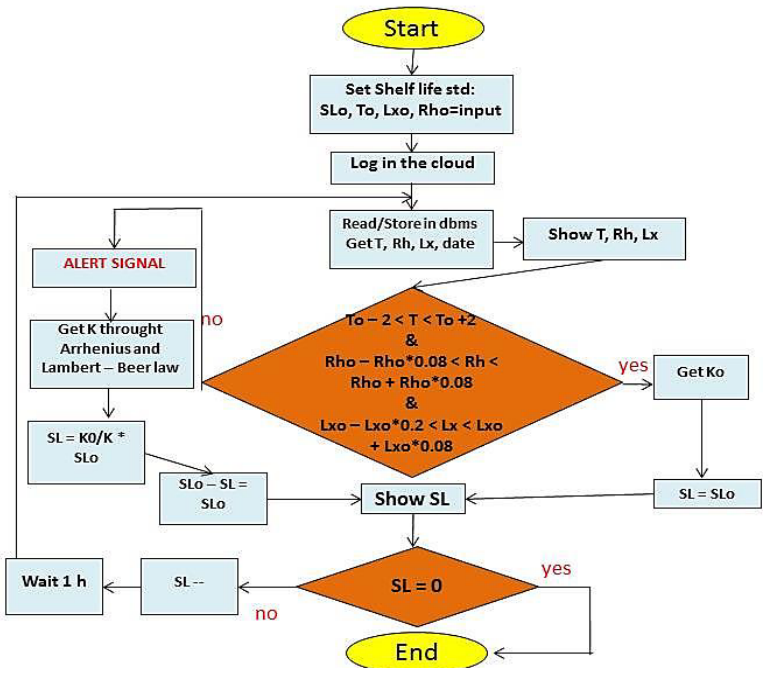

Figure 6. Shelf life estimation flow chart

First step: Store input data about standard shelf life (SLo - inserted in hours), temperature (To), Humidity (Rho) and illuminance (Lxo) of reference. Acquire from the WSN information about real time $\mathrm{T}, \mathrm{Rh}, \mathrm{Lx}$ than show them.

Second step: Verify if the current conditions are significantly far away from the standard ones (the chosen range is due to sensors performances). If not, calculate the Ko. The SL is the same as the SL standard (stored in input).

Third step: If the product is not in the standard condition, create an alert signal. Than calculate the degradation speed coefficient (K) through Arrhenius (1) and estimate the new shelf life as a fraction of the standard one: the new shelf time is calculated using the ratio between the current degradation speed and the previous one. A database in which all the required constant values are stored is needed. Finally set the new SL as a maximum limit: in preventive view and the SL cannot be increased (even if returning in the standard conditions).

Forth step: Show remaining shelf time and repeat the loop every hour.

Considering that the temperature values have an accuracy of $+1-2^{\circ} \mathrm{C}$ we can reasonably assume that the new value of the rate of degradation $\mathrm{K} 1$ is to be understood between a K1- and $\mathrm{K} 1+$ evaluated according to the Arrhenius law (1), respectively, at $\mathrm{T} 1+2{ }^{\circ} \mathrm{C}$ and to $\mathrm{T} 1-2^{\circ} \mathrm{C}$. Therefore the shelf time is valid in an interval between ts1and ts1+, obtained from eq.(3), using respectively K1- and $\mathrm{K} 1+$.

\section{CONCLUSIONS}

In this paper a monitoring system of a perishables goods supply chain, made up by the combination of a WSN and a further data processing for shelf life prediction, has been presented. The proposed approach is advantageous and suitable for a low cost implementation and allows at the same time a reduction of the waste in the supply chain by a right evaluation of the product life and an improvement of food safety, as well as food organoleptic qualities. Our work was therefore just a demonstration of feasibility, but future prospectives are even more compelling: the expansion of the sensor network using $\mathrm{PH}, \mathrm{O} 2, \mathrm{CO} 2$, ethylene, shock and other appropriate sensors, the implementation of a GPS system (for tracking info) and of a GSM communication (that make the gateway unnecessary), the development of a dedicated app, are all useful and achievable option for improving the monitoring approach and for implementing an environmental control system.

\section{REFERENCES}

[1] FAO/WHO. "FAO/WHO guidance to governments on the application of HACCP in small and/or less-developed food businesses",14 October 2007.

[2] ISO 22000 FSMS 2005

[3] Espenson ,J.H. "Chemical kinetics and reaction mechanisms”. Mc Graw-Hill

[4] Labuza, T.P. “Application of chemical kinetics to deterioration of foods. Journal of Chemical Education” 61, 348-358.

[5] Fu, B. , Labuza, T.P. "Shelf life prediction: theory and application. Food Control."

[6] S. Hoppough, “Shelf Life,” Forbes, Apr. 24, 2006.

[7] Dargie, W. and Poellabauer, C., "Fundamentals of wireless sensor networks: theory and practice", John Wiley and Sons, 2010.

[8] Sohraby, K., Minoli, D., Znati, T. "Wireless sensor networks: technology, protocols, and applications", John Wiley and Sons, 2007

[9] Adel S. Sedra, Kenneth C. Smith "Microelectronic Circuits", 6th Edition. 